\title{
La experiencia de conexión existencial como concepto naturalista de espiritualidad ${ }^{1}$
}

\author{
Ricardo Braun \\ Universidad de Lima \\ Grupo Interdisciplinario de Investigación Mente y Lenguaje \\ Pontificia Universidad Católica del Perú
}

\begin{abstract}
Recibido: 20 de mayo del 2017 / Aceptado: 8 de junio del 2017
doi: https://doi.org/10.26439/persona2017.n20.1741
\end{abstract}

Este artículo propone la incorporación del término experiencia de conexión existencial como un candidato para reemplazar al de espiritualidad, a fin de evitar las connotaciones religiosas y representar mejor una visión naturalista de la experiencia humana. Se discute el debate actual acerca del término que mejor represente el fenómeno asociado a las experiencias de conexiones del sujeto con su propia existencia, y una existencia situada en relación con el resto de elementos del universo. En este sentido, el vínculo con los elementos y seres que lo rodean genera un sentimiento de pertenencia y compromiso con la realidad. Asimismo, el término existencial alude a los temas abordados tradicionalmente por la filosofía existencialista.

espiritualidad / naturalismo / experiencia existencial

\section{The Experience of Existential Connection as a Naturalistic Concept for Spirituality}

In this paper, I propose the use of the term existential connection experience as a possible replacement of the term spirituality, to avoid religious connotations and to better represent a naturalistic fuller view of human experience. I discuss the current debate on the best term to represent the phenomenon associated to connections of the human subject with his/her own existence and their situated existence with the universe. This term, which should become a concept represents the relationship of belonginess and commitment with reality. Moreover, the existential component refers to the traditional topics dealt with by philosophical existentialism.

\section{spirituality / naturalism / existential experience}

Correo electrónico: rbraun@ulima.edu.pe

1 Agradezco al Instituto de Investigación Científica (IDIC) de la Universidad de Lima por el apoyo en el proyecto de investigación sobre la naturalización de la filosofía y psicología moral, del que este artículo forma parte. Agradezco también a mi asistente de investigación del IDIC, Fernando García Blesa, que colaboró en la elaboración y reflexión de este ensayo. 


\section{INTRODUCCIÓN}

Una legítima preocupación en el desarrollo de la ciencia de la psicología es la búsqueda de la mejor comprensión de la experiencia humana. La historia de la disciplina, si la tomamos en el sentido convencional en sus inicios, con los trabajos de Wundt a finales del siglo XIX, revela la constante controversia alrededor del objeto de estudio, la metodología, la visión de ciencia, el vocabulario $\mathrm{y}$, por supuesto, las presuposiciones metafísicas y epistemológicas alrededor de estos aspectos.

En efecto, la historia de la vida académica de Wundt, poco enseñada a los futuros profesionales y científicos de la psicología, permite ejemplificar la mencionada preocupación. Usualmente, se presenta a Wundt como el iniciador de la psicología experimental y se le muestra como un pionero de esa metodología en la incipiente psicología científica. Ciertamente eso es correcto, pero quiero señalar, más bien, los problemas que lo acercan a la reflexión que desarrollo en este artículo.

En su época, la visión secular del mundo parecía convivir, por un lado, con ideas espiritualistas asociadas a la religión convencional, pero también, por otro lado, con prácticas y suposiciones de corte ocultista. El dualismo como concepción humana era una visión estándar, de acuerdo con la creencia cristiana de la supervivencia anímica después de la muerte.

Leipzig, la ciudad donde Wundt fue contratado como profesor, era conocida por su actitud antimodernista y su gusto por ciertas formas de ocultismo. De hecho, existía una casa editorial especializada en literatura espiritualista y ocultista, la Oswald Mutze Verlag, que promovía la publicación de trabajos de quienes consideraba "espiritistas de avanzada" (Kohls y Benedikter, 2010, p. 45). La Universidad de Leipzig tampoco era ajena a esta actitud benevolente hacia el espiritismo en el sentido más radical. El joven profesor Wundt tuvo que enfrentar una hostilidad inesperada.

Habiendo invitado la universidad a un médium espiritista para que hiciera ciertas demostraciones de contacto con los muertos, Wundt (1879) reaccionó a estos supuestos experimentos con una feroz crítica escrita, en la que denunciaba desde problemas metodológicos hasta la posibilidad de fraude del médium. La respuesta de la administración universitaria no se hizo esperar. Wundt fue hasta demandado legalmente por dañar la reputación de los académicos que habían organizado estas presentaciones. Cómicamente, fue también acusado de estar poseído por un espíritu maligno cuando escribió su ataque a los espiritistas. A la muerte de algunos de los entusiastas espiritistas delauniversidad, el presupuesto de sus sueldos pasó a ser ofrecido al mismo Wundt para que desarrollara el Institut für Experimentelle Psychologie, considerado por los historiadores de la psicología una de las instituciones pilares en la creación de la psicología científica.

Este breve recuento del periodo inicial del profesor Wundt tiene por objeto señalar el problema, desde los orígenes de la psicología moderna, en cuanto a las 
concepciones dualistas y espiritualistas de tradición más bien religiosa, que entraban en conflicto con una teoría científica de la mente humana de corte naturalista, en concordancia con una visión más unificada de la realidad. Separar la visión, digamos de la física, de la concepción de los humanos como algo demasiado distinto, y en algunas suposiciones casi opuestas a la primera, hacía el trabajo del desarrollo de la psicología científica una tarea con obstáculos constantes. Y eso se demuestra en las críticas de personajes en la historia de la psicología de los últimos cien años a los conceptos asociados a creencias religiosas o a prácticas también asociadas a las religiones tradicionales.

En este trabajo, quiero explorar el concepto de espiritualidad como una reflexión preliminar para el desarrollo de un futuro concepto que pueda ser utilizado en forma usual en la teoría y práctica de la ciencia de la conducta humana. La psicología tiene por objeto entender la experiencia humana, y esta no puede ser comprendida sin incorporar de forma científica el fenómeno que se asocia con el término espiritualidad, aunque no siempre con un concepto unívoco. Por ello, considero que es una tarea relevante abordar esta problemática. Como dice Armstrong (1999) elocuentemente:

Nuestra cultura científica nos educa para enfocarnos en el mundo físico y material que aparece ante nosotros. Este método de observar el mundo ha logrado grandes resultados. Una de sus consecuencias, sin embargo, es que hemos, por así decirlo, eliminado el sentido de lo "espiritual" o lo "sagrado" que impregna las vidas de personas en sociedades más tradicionales a todo nivel, y que fue en su momento un componente esencial en la experiencia humana del mundo. (p. 10)

\section{ReducCiONISMO CIENTíFICO}

El reduccionismo es tanto una visión metafísica como metodológica en la ciencia. No discutiré el concepto que he revisado y cuya problemática he mostrado anteriormente (Braun, 2006, 2008). Sin embargo, sí quiero señalar las tres fuentes clásicas del reduccionismo en el concepto de espiritualidad, como ejemplos de actitudes científicas que limitan el posible estudio de un fenómeno existente.

En breve, todo reduccionismo implica una forma explicativa que intenta comprender la naturaleza de las cosas reduciendo la complejidad a sus formas más simples, a fenómenos en último término fundamentales. La filosofía de la ciencia ofrece elocuentes ejemplos de reduccionismos exitosos, pero también de reduccionismos no solo limitantes, sino engañosos: al final, no somos sino partículas subatómicas gobernadas bajo el imperio de las leyes inexorables de la naturaleza.

La postura optimista del reduccionismo tuvo repercusión obvia en la naciente ciencia psicológica e inclusive en corrientes no tan reduccionistas; así, se puede percibir la influencia de la vena positivista de principios del siglo $\mathrm{xx}$. El problema que se puede ver en la actitud en contra del estudio de la espiritualidad como fenómeno es la asociación 
espiritualidad $=$ religiosidad. Por ello, no sorprende que cualquier referencia a lo espiritual en las visiones reduccionistas corresponda a explicaciones acerca de las creencias religiosas y sus correspondientes comportamientos y cosmovisiones. Y, por lo tanto, las conclusiones negativas a las que arriban los críticos no serían aplicables a quienes afirman tener experiencias espirituales sin ser experiencias religiosas, y menos confesionalmente religiosas.

Así, Freud en El porvenir de una ilusión (1988 [1927]) reconoce la fuerza interna que producen las ideas religiosas y habría que indagar su eficacia causal, aun cuando estas sean irracionales. Es más, dado que concibe la creencia en una divinidad como una proyección de la figura paterna, y no como la creencia de algo real, lleva estas ideas a una forma de neuroticismo.

Cattell (1939) representa otra forma de reduccionismo, en la cual el fenómeno espiritual es considerado como una tonta superstición. Coincide con Freud en que este es un fenómeno que actúa como una poderosa fuerza en las vidas de las personas. Con una vena conductista, trató de indagar las bases motivacionales para las actitudes religiosas y concluyó que la religión era una motivación para evitar el miedo, para proteger a otros y ser protegido. Esta visión debe de haber influido en la concepción skinneriana posterior, en la que se observa una vena motivacional similar en la explicación reduccionista de la espiritualidad, como forma de adquisición de supersticiones vía condicionamientos operantes.
Finalmente, cabe mencionar a James (1985 [1902]), que en una concepción más bien pragmatista $-\mathrm{y}$ por ello reduccionista - plantea que las creencias religiosas son útiles y realmente no tiene sentido cuestionarlas, en tanto funcionen adecuadamente. La espiritualidad merece ser estudiada porque ha sido probada como beneficiosa. Este tipo de reduccionismo aparece sutilmente, puesto que pareciera que se reconoce el fenómeno, pero es objeto de estudio por sus efectos, no por la experiencia misma.

\section{El PROBLEMA DEL TÉRMINO}

\section{ESPIRITUALIDAD}

Como se advirtió anteriormente, la casi ecuación entre espiritualidad y religiosidad provoca la problemática del estudio más extensivo del fenómeno al que se quiere aludir. El razonamiento más simple y falaz sería que, sin religiosidad, no se puede tener una genuina experiencia de espiritualidad. $\mathrm{Y}$ es por esta razón que el término debe ser revisado y actualizado para desvincularlo de la supuesta relación necesaria con las actitudes y creencias religiosas.

De acuerdo con su origen etimológico, espiritualidad proviene del término latino spiritus que significa "aliento o respiro", y a su vez del griego $\pi v \varepsilon \tilde{v} \mu \alpha$ (рпеита), el aliento vital de los seres vivos, que cesa o "expira" con la muerte. Se trata, por tanto, del principio vital que anima a todo ser vivo, lo que incluye a lo corpóreo. Desde el racionalismo, el espíritu es aquella cosa pensante, res cogitans cartesiana, separable del cuerpo (García, 2010). 
En el idealismo hegeliano, el espíritu es aquella Idea separada del cuerpo que se aliena en la naturaleza y en los individuos a manera de espíritus individuales, que se orientan hacia el Espíritu Absoluto, que él denominaba como verdad. Posteriormente, las corrientes más contemporáneas han utilizado la palabra espíritu para referirse a aquellas cualidades como la conciencia, la libertad y la intencionalidad (García, 2010).

Luego de revisar las diversas acepciones, no resulta extraño que el término haya generado resistencia conceptual en aquellas personas que no creen en la existencia de seres u objetos inmateriales, como el espíritu o alma. Por lo tanto, en el contexto no religioso se generan posiciones distintas, y en algunos casos opuestas, sobre el uso del término espiritualidad.

Si bien algunos utilizan el término espiritualidad secular, muchas personas no definirían su pensamiento como una forma de "espiritualidad", sino como la cultivación consciente de la reflexividad y su preocupación por el estilo de vida (Rossiter, 2014). En contraste, Battistelli (2014) rechaza el uso de la palabra espiritualidad porque hace referencia a ideas distintas que parten del reconocimiento de la interconexión de todos los elementos del universo, las cuales pueden ser discutidas de manera separada utilizando conceptos que no aludan a lo sagrado, como paz, maravilla, asombro, conexión con el universo, entre otros. Para este autor, no hay nada que requiera ser trascendido en una existencia finita, a la que nosotros mismos le damos un significado.
Sin embargo, encontramos también defensores del término espiritualidad secular. Walach la define como una forma o modo de conciencia, cuyas experiencias de conexión ocurren a través de la inspiración artística o científica. Es más, sostiene que la espiritualidad y la ciencia son compatibles, pues hay dualidad, pero no dualismo (Grosso, 2015).

Otro defensor del término es el filósofo y neurocientífico Sam Harris (2012, 2014), que promueve la desvinculación de la religión con el concepto de lo espiritual. A pesar de la problemática asociación de la palabra espiritual con su etimología, Harris afirma que no hay otra opción para representar este fenómeno, pues, por ejemplo, un término como místico sería aún más oscuro, y otro como contemplativo sería muy restrictivo. En esta misma línea, Rosch (2012) afirma que los ateos también pueden ser espirituales, puesto que la espiritualidad es la sensación de conexión profunda entre las personas y con el universo en general.

\section{¿POR QUÉ PROPONER UN TÉRMINO}

\section{ALTERNATIVO? ¿ES EL LENGUAJE}

TAN IMPORTANTE?

El lenguaje tiene una función esencial en las representaciones y construcciones de la realidad. Como afirma el filósofo John Searle (1995), el lenguaje es un elemento constitutivo de los hechos sociales. Si se admite que la espiritualidad es un hecho tanto individual como social, el término espiritualidad no religiosa encierra una gran contradicción: se emplea un lenguaje metafísicamente dualista para 
hacer referencia a un fenómeno metafísicamente monista.

Por lo tanto, es necesario contar con un término naturalista para hacer referencia a una realidad natural; en cambio, lo desarrollado por la espiritualidad teísta o mística habla de un fenómeno ontológicamente distinto e incluso contrario al no religioso: este conjunto de acontecimientos agrupados es originado, fundamentado y protagonizado por uno o más seres inmateriales que intervienen en la realidad material, haciendo posible las experiencias de sentido, asombro e interconexión. En contraste, para una cosmovisión de carácter religioso, sin la existencia de un espíritu (posiblemente inmaterial), no hay espiritualidad posible. En consecuencia, es necesario proponer un término que remita al fenómeno psicológico en forma naturalista y que no requiera la referencia a realidades espirituales y religiosas.

\section{LA PROPUESTA: CONEXIÓN EXISTENCIAL}

El término que se propone, por lo menos en forma preliminar, es conexión existencial. Este concepto abarcaría el fenómeno subjetivo en el que el ser humano contempla y se conecta con su propia existencia con asombro y reverencia.

El concepto de conexión implica el vínculo del sujeto con su propia existencia, una existencia situada en relación con el resto de elementos del universo. En este sentido, el vínculo con los elementos y seres que lo rodean genera un sentimiento de pertenencia y compromiso con la realidad.

Asimismo, el término existencial alude a los temas inicialmente abordados por la filosofía existencialista: reflexiones sobre la realidad, la conciencia, el sentido de vida, la muerte y la libertad (Sartre, 1999). Sobre este término, Gardner (2014) reflexiona acerca del concepto de inteligencia existencial, propia de aquellos individuos que exhiben la tendencia a cuestionarse sobre condiciones de la existencia humana, como la vida, la muerte, las realidades últimas y ciertas experiencias profundas de amor o asombro por una obra de arte. No la conceptualiza como una meta de apropiación de una verdad final, sino como la capacidad de la especie por interesarse en las cuestiones de su propia existencia, apreciada en todas las culturas y distintiva del ser humano. Dice Gardner (2014): "Mi conclusión es que la variedad de inteligencia espiritual, que he definido de una manera restringida y que he denominado 'existencial', puede ser aceptable, y que la 'inteligencia espiritual', en su definición más amplia, no lo es" (p. 89).

En suma, emplearemos el concepto de experiencia de conexión existencial, en reemplazo de espiritualidad, y por economía del lenguaje, abreviaremos conexión existencial, por experiencia existencial. Finalmente, nos referiremos a las escalas psicométricas de inteligencia espiritual como escalas de inteligencia existencial. 


\section{COMPONENTES DE LA CONEXIÓN EXISTENCIAL}

Actualmente, se pueden encontrar definiciones muy diversas de este fenómeno, incluso con puntos claramente contradictorios entre sí. Sin embargo, se hallan ciertos conceptos transversales a todas las perspectivas y estilos de abordaje a este fenómeno. En otras palabras, los conceptos que a continuación presento serían componentes de la conexión existencial.

\section{Conciencia}

Involucra la autopercepción de la inmediatez de la experiencia: la capacidad de atender el aquí y el ahora, para entrar en un estado de flujo que involucre una apertura a la dinámica del progreso ante problemas aparentemente irresolubles (Wright, 2000). Asimismo, requiere que el sujeto sea consciente de sí mismo (Zohar y Marshall, 2000). Esta característica fue incluida en una escala de inteligencia espiritual desarrollada por Kumar y Mehta (2011).

\section{Búsqueda de sentido}

La experiencia de conexión existencial moldea la identidad y orienta a abordar las preguntas sobre el sentido y el propósito de vida (Wright, 2000): cuestiones asociadas a las condiciones de la existencia humana, como la muerte, la relación con los demás, con la naturaleza, entre otras. Asimismo, el propósito de vida es otra de las dimensiones/factores de la escala de Kumar y Mehta (2011).

Sobre el sentido, Tillich (1980) desarrolla el término de preocupación final (ultimate concern), que alude a la propia construcción y respuesta ante la pregunta del sentido de vida. Una de sus principales características es que la persona se entrega totalmente como sujeto hacia el objeto de su preocupación. Además, sostiene que involucra a la totalidad de la personalidad, que va más allá de la racionalidad —si por ello entendemos la habilidad de desarrollar argumentos válidos en el sentido clásico-y que nos permite pensar más allá de la temporalidad.

\section{Lo sagrado}

Sobre este tema, Otto (1980) afirma que la experiencia de lo sagrado es difícil de explicar, imposible de enseñar y básica en todas las religiones. Para referirse a la experiencia de lo sagrado, habla de mysterium tremendum, que es la relación con una otredad completa, que asombra y desconcierta. La reacción mental ante este misterio es el estupor, un estado de gran asombro que nos deja atontados.

Es importante aclarar que lo sagrado no se limita a las concepciones tradicionales de Dios, de poderes superiores o de divinidades. Creencias, prácticas, experiencias, relaciones, motivaciones, arte, naturaleza, guerra y cualquier parte de la vida, positiva o negativa, pueden ser significadas como sagradas (Zinnbauer, Pargament y Scott, 1999).

\section{Trascendencia}

Esteconceptoresultaseralgoproblemático para modelos no religiosos, pues ha sido asociado con lo divino: el alma o espíritu va más allá de, trasciende, la existencia 
material del cuerpo. Sin embargo, el concepto también es incorporado en perspectivas no religiosas. Wright (2000), por ejemplo, la define como orientación a maravillarse y asombrarse sobre el misterio del universo, que implica una apertura a la estimulación a través de una imaginación activa.

Un término que podría aclarar el panorama es el concepto de lo extático. En este sentido, calificar de extática a la conexión existencial implica que este fenómeno abarca más de una dimensión de la personalidad humana. La experiencia extática se orienta más allá de la inmediatez del momento. El ejemplo del amor utilizado por Tillich (1980) resulta bastante ilustrador. El amor tiene un componente emocional, mas no es solo emociones, pues involucra la totalidad de la personalidad y hace que la persona enamorada vaya más allá de su egocentrismo inmediato para preocuparse por alguien que demanda lealtad $y$ compromiso. En el caso de la conexión existencial, el compromiso es con uno mismo y los demás, así como con los valores que derivan de esta experiencia.

\section{Interconexión}

Por otro lado, el holismo y la interconexión con los demás son conceptos centrales cuando se trata de definir la conexión existencial. Dentro de la práctica existencial, se promueve un nuevo modo de percibir los fenómenos, una visión holística que integre y trascienda los límites existentes del conocimiento, que reconozca que todos estamos envueltos en el proceso cíclico de la naturaleza (Maxwell, 2003). Esta capacidad está presente en la propuesta del pensamiento unificador de Zohar y Marshall (2000), aquel que nos permitesercreativos, reflexivos eintuitivos.

\section{Conexión religiosa y no religiosa}

\section{Conexión existencial religiosa o teísta}

La conexión religiosa o teísta aborda los ejes centrales mencionados en la sección anterior, desde la creencia en uno o más seres inmateriales que intervienen $y$ explican la realidad. Estos seres (dioses, espíritus, energías) son los que dan sentido y propósito, los que trascienden todos los objetos y fenómenos materiales, y permiten al sujeto encontrar esa interconexión por medio de la experiencia mística o religiosa.

Veamos una definición teísta de inteligencia espiritual. Safara y Bhatia (2013) la definen como aquella involucrada en la vida interna de la mente (es decir, como espíritu) y su relación con el ser en el mundo. Implica la capacidad de entender profundamente las preguntas existenciales, un insight en múltiples niveles de conciencia $\mathrm{y}$, por último, una conciencia de la mente (espíritu) como el terreno del ser o como la fuerza creativa viva de la evolución (Hood, Hill, y Spilka, 2009).

\section{Conexión existencial no religiosa}

La conexión existencial no religiosa representa lo que las personas hacen para alcanzar un conjunto de objetivos, como el sentido de vida, la totalidad, la 
interconexión con los demás, la verdad y el potencial interno (Zinnbauer, Pargament y Scott, 1999). Asimismo, para Hahn (citado en Hyland, 2013), debe basarse en evidencia corroborable, no en creencias sin algún fundamento que pueda ser contrastado en la realidad (p. 249).

Una definición no religiosa de inteligencia existencial resulta de mucha utilidad para efectuar la comparación con la perspectiva religiosa trabajada anteriormente. La esencia de esta inteligencia sería la capacidad de realizar el potencial propio para cumplir un objetivo de servicio a la humanidad en un sentido fraterno; se trata de lo que diferencia al ser humano de un robot: la facultad de definir el propio sentido de vida y de ser compasivo con los demás seres que lo rodean (Kumar y Mehta, 2011). En la prueba que proponen estos autores, la inteligencia existencial tiene seis factores: propósito en la vida, valores humanos, compasión, compromiso con la humanidad, un self comprensivo y conciencia.

\section{Conclusión}

He querido presentar una propuesta que, con los obvios desafíos que presenta, permita promover los estándares adecuados de la rigurosidad científica ante un fenómeno existente que trasciende el mundo de los creyentes religiosos y que, obviamente, los incluye. Además, como toda meta científica, debe buscar enriquecer y profundizar en el objeto de estudio. Una visión naturalista, no reduccionista, es nuestra mejor herramienta en una era en la que no podemos tener fronteras disciplinarias o sectarias. Las neurociencias, la sociología, la psicología, la lingüística y otras nos permitirán entender mejor este fenómeno si lo hacemos más incluyente y menos restrictivo.

Los conceptos que pueden volverse definiciones operacionales más unívocas son más eficientes en el trabajo psicológico. Recordemos que una buena teoría es necesaria para proporcionar un marco de referencia para interpretar y comprender mejor nuestros hallazgos. Nunca está demás subrayar la importancia de la claridad conceptual, más aún en el complejo tema de esta breve reflexión. Espero que el concepto de conexión existencial pueda servir como una modesta propuesta.

\section{Referencias}

Armstrong, K. (1999). A History of God. Londres: Vintage Books.

Battistelli, T. (28 de agosto del 2014). A Humanism without the word 'spirituality'. Recuperado dehttp://humanistlife. org.uk/2014/08/28/a-humanismwithout-the-word-spirituality/

Braun, R. (2006). La situación de la teoría y terminología de la psicología popular y el futuro de la psicología científica. Persona, 9, 77-94.

Braun, R. (2008). El eliminativismo filosófico y su ataque a la psicología. Persona, 11, 51-67.

Cattell, R. B. (1939). Psychology and the Religious Quest. Londres: Thomas Nelson and Sons. 
Freud, S. (1988 [1927]). El porvenir de una ilusión. Buenos Aires: Amorrortu.

García, J. (2010). Espíritu. En Á. González (Ed.), Diccionario de filosofía (pp. 389-393). Navarra: Ediciones Universidad de Navarra.

Gardner, H. (2014). ¿Hay más inteligencias? En La inteligencia reformulada: las inteligencias múltiples en el siglo XXI (pp. 84-89). Barcelona: Paidós.

Grosso, M. (2015). Secular Spirituality: The Next Step Towards Enlightenment. Journal of Scientific Exploration, 29(2), 356-360.

Harris, S. (27 de junio del 2012). In Defense of "Spiritual" [Mensaje en un blog]. Recuperado de https://www. samharris.org/blog/item/a-plea-forspirituality

Harris, S. (2014). Waking Up: A Guide to Spirituality Without Religion. Nueva York: Simon \& Schuster.

Hood, R., Hill, P., y Spilka, B. (2009). The Psychology of Religion: An Empirical Approach. Nueva York: Guilford.

Hyland, T. (2013). Buddhist Practice and Educational Endeavour: In Search of a Secular Spirituality for StateFunded Education in England. Ethics and Education, 8(3), 241-252. doi: 10.1080/17449642.2013.876791

James, W. (1985 [1902]). The Varieties of Religious Experience. Cambridge, MA: Harvard University Press.

Kohls, N., y Benedikter, R. (2010). The Origins of the Modern Concept of "Neuroscience". En J. Giordano y B.
Gordijn (Eds.), Scientific and Philosophical Perspectives in Neuroethics. Cambridge: Cambridge University Press.

Kumar, V., y Mehta, M. (2011). Scale for Spiritual Intelligence (SSI). PsycTESTS. doi: 10.1037/t16725-000

Maxwell, T. P. (2003). Integral Spirituality, Deep Science and Ecological Awareness. Zygon: Journal of Religion and Science, 38(2), 257-276. doi: 10.1111/1467-9744.00499

Otto, R. (1980). The experience of the Holy. En D. Stewart (Ed.), Exploring the Philosophy of Religion (pp. 20-33). Nueva Jersey: Prentice Hall.

Rosch, S. (3 de marzo del 2012). Atheists Can Be Spiritual Too [Mensaje en un blog]. Recuperado de http://www. huffingtonpost.com/staks-rosch/atheistscan-be-spiritual_b_1316619.html

Rossiter, G. (2014). Decoding the miseen-scène of contemporary secular spirituality: a research monograph. Part I: context, approach and relevant literature. Journal of Religious Education, 62(2), 65-78. doi: 10.1007/ s40839-014-0008-Z

Safara, M., y Bhatia, M. (2013). Spiritual Intelligence. Delhi Psychiatry Journal, 16(2), 412-423.

Sartre, J. P. (1999). El existencialismo es un humanismo. Barcelona: Edhasa.

Searle, J. (1995). The Construction of Social Reality. Londres: Penguin Philosophy.

Tillich, P. (1980). The experience of ultimate concern. En D. Stewart (Ed.), Exploring 
the Philosophy of Religion (pp. 9-19). Nueva Jersey: Prentice Hall.

Wright, A. (2000). The psychology of spiritual experience. En Spirituality and Education (pp. 36-46). Nueva York: Routledge.

Wundt, W. (1879). Der Spiritismus: Eine sogenannte wissenschaftliche Frage. Leipzig: Engelmann.
Zinnbauer, B. J., Pargament, K. I., y Scott, A. B. (1999). The Emerging Meanings of Religiousness and Spirituality: Problems and Prospects. Journal of Personality, 67(6), 889-919.

Zohar, D., y Marshall, I. (2000). SQ Spiritual Intelligence: The Ultimate Intelligence. Recuperado de http:// www.alisonmorgan.co.uk/Books/ Zohar\%202000.pdf 
